
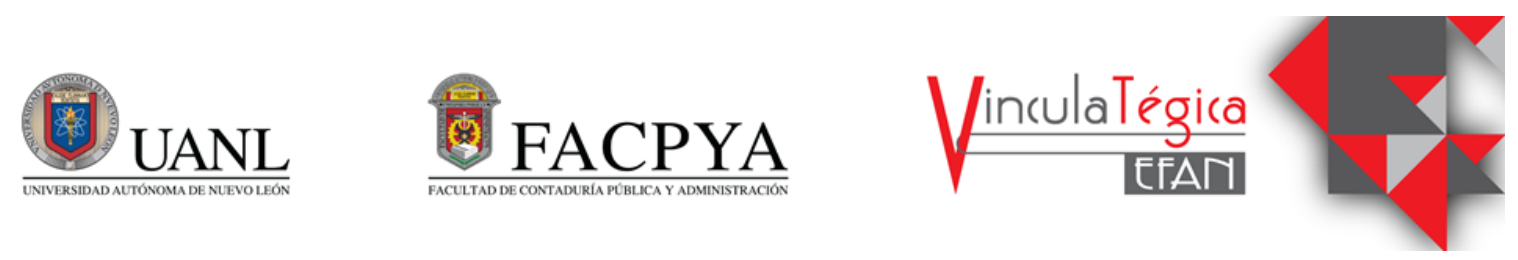

\title{
El capital humano y su relación con el desempeño en escuelas de Educación Media Superior
}

\author{
América Lorena González Cisneros ${ }^{1}$ y Norma Angélica Pedraza Melo 2 \\ ${ }^{1}$ Universidad Autónoma de Tamaulipas (UAT), gonzalezcisnerosamericalorena@gmail.com, Centro \\ Universitario Adolfo López Mateos, 8341263223 \\ ${ }^{2}$ Universidad Autónoma de Tamaulipas (UAT), correloelectrónicodelautor@correo.com, Centro \\ Universitario Adolfo López Mateos, 8341481880
}

Información del artículo revisado por pares

Fecha de aceptación: junio-2021

Fecha de publicación en línea: diciembre-2021

DOI: https://doi.org/10.29105/vtga7.1-159

\begin{abstract}
Resumen
El estudio analiza la relación entre el capital humano $(\mathrm{CH})$ y el desempeño organizacional (DO) en las instituciones tecnológicas de educación media superior (EMS) en el estado de Tamaulipas, México. A través de un diseño no experimental, cuantitativo y explicativo, se aplicó un cuestionario a una muestra no probabilística de 300 docentes del sector. A través del análisis factorial exploratorio (AFE) se determinó la estructura subyacente de las variables latentes y por medio de SEM (modelado de ecuaciones estructurales) se validaron las hipótesis. Los hallazgos detectados indican que las dimensiones del $\mathrm{CH}$ tienen un efecto positivo y significativo en el DO, a excepción de las competencias docentes; esto le permite a la gerencia educativa de estos planteles establecer estrategias para la gestión de su $\mathrm{CH}$ como un recurso indispensable de su función.
\end{abstract}

Palabras clave: capital humano; desempeño organizacional; instituciones tecnológicas; educación media superior.

Códigos JEL: J24, M12, M54

\section{Abstract}

The study analyzes the relationship between human capital $(\mathrm{CH})$ and organizational performance (OD) in technological institutions of upper secondary education (EMS) in the state of Tamaulipas, Mexico. Through a nonexperimental, quantitative and explanatory design, a questionnaire was applied to a non-probabilistic sample of 300 teachers from the sector. Through exploratory factor analysis (EFA) the underlying structure of the latent variables was determined and through SEM (structural equation modeling) the hypotheses were validated. The findings detected indicate that the dimensions of $\mathrm{CH}$ have a positive and significant effect on OD, with the exception of teaching skills; This allows the educational management of these schools to establish strategies for the management of their $\mathrm{CH}$ as an indispensable resource of their function.

Key words: human capital; organizational performance; technological institutions; upper secondary education.

JEL Codes: J24, M12, M54 


\section{INTRODUCCIÓN}

La educación media superior (EMS) en su modalidad tecnológica, es actualmente una de las opciones mas elegidas por los jóvenes para cursar sus estudios de bachillerato (OCDE, 2017). Entre sus retos, ha sido enlazar los conocimientos del aula con los requerimientos solicitados en el sector (OCDE, 2018) al mismo tiempo de establecer sinergia entre sus recursos tangibles (infraestructura, equipamiento, entre otros) e intangibles (actores internos y externos, gestión, desempeño, entre otros) a fin de lograr beneficios que se traduzcan en un mejor desempeño educativo a nivel regional y nacional (Araya-Castillo y Rivera-Arroyo, 2021).
En este sentido, el interés por los recursos educativos intangibles como: $\mathrm{CH}$, gestión de conocimientos, desempeño organizacional (DO), aprendizaje organizacional, entre otros, empiezan a ser considerados como una fuente de desarrollo para las instituciones educativas (OCDE, 2017; Segredo, 2016). Sin embargo, existen pocos estudios tanto teóricos como empíricos tratando temas intangibles en el contexto de la EMS (Cabrol y Székely, 2012; Farah y Abouzeid, 2017; González-Loureiro y Teixeira, 2015; Guthrie y Dumay, 2015; López, 2016; OCDE, 2017; Orozco, 2014; Pérez y Pino, 2018; Rondón y Ammar, 2016).

$\mathrm{Al}$ respecto, la figura 1, muestra la producción de investigaciones generadas en la Web of Science (WoS) relacionadas con el $\mathrm{CH}$ educativo de 2015 a la fecha:

Figura 1: Publicaciones científicas de $\mathrm{CH}$ en instituciones educativas públicas

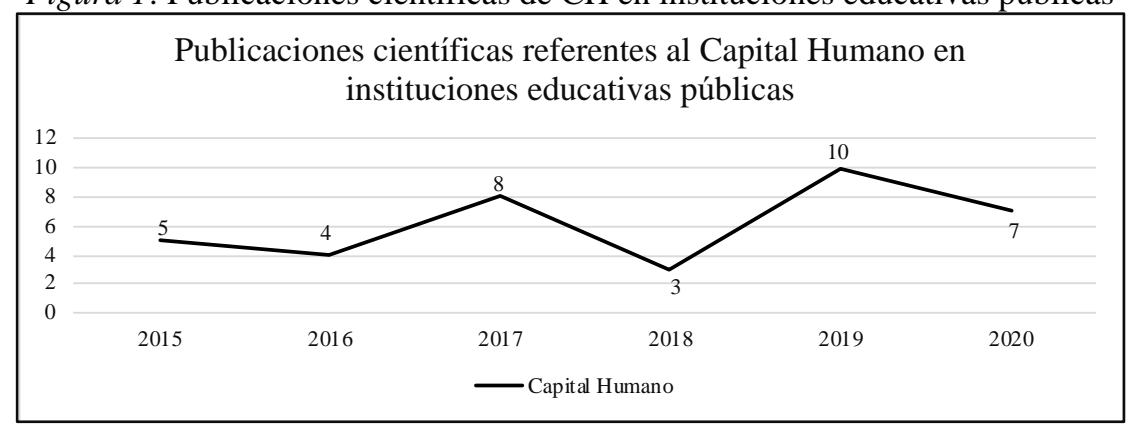

Fuente: elaboración propia en base a WOS, 2021.

La figura 1 , muestra 37 publicaciones en un periodo de cinco años (2015-2020), lo que denota evidentemente una falta de exploración en el tema. Por tanto, se estiman necesarias más investigaciones en el contexto, relacionadas con variables intangibles como el CH (Bak, 2012), pues su impacto, permite generar valor educativo otorgando beneficios en el desempeño de estas instituciones (Chatterji y Kiran, 2017). Así, el objetivo de la presente investigación ha sido analizar la variable $\mathrm{CH}$ y su influencia en el $\mathrm{DO}$ en el marco de las instituciones de EMS tecnológica.

\section{MARCO TEÓRICO}

\subsection{El desempeño organizacional en} instituciones de EMS tecnológica

El bachillerato técnico, constituye un bloque fundamental de formación para los jóvenes en muchos países; en esta etapa, la comunidad estudiantil busca su integración a la educación superior o en su caso, satisfacer la demanda del mercado laboral (OCDE, 2017). Sin embargo, este esquema educativo, ha sido minimizado por aspectos políticos y ha sido opacado por la popularidad del bachillerato general (OCDE, 2017).

Para Machorro y Romero (2017), el estudio del DO en el contexto educativo ha sido un tema complicado por la cantidad y diversidad de actores que influyen y porque los resultados escolares, se han enfocado únicamente en tratar índices como: ingreso/egreso estudiantil, infraestructura, planes de estudios, equipamiento, entre otros (Agasisti et al., 2016; Bueno et al., 2008; Sánchez et al., 2009; Segatto y Abrucio, 2017; Vidal, 2017; Wu et al., 2012). En este sentido, casi toda América Latina y el Caribe ha enfrentado índices negativos de DO relacionados con la falta de pertinencia y calidad educativa (Cabrol y Székely, 2012), lo que repercute en problemas de roles escolares e índices de deserción elevados (OCDE, 
2017).

Aunado a lo anterior, los modelos de estudio de DO en instituciones educativas han sido escasos, complejos y controversiales (Machorro y Romero, 2017) y las dimensiones utilizadas por los autores no han sido consensuadas. En el tema, Mertens quien realizó uno de los primeros trabajos, identificó al gasto educativo (costo por estudiante), capacidad educativa (infraestructura y recursos) y rendimiento estudiantil (promedio por estudiante), como algunos indicadores de la variable (Mertens, 1979). La tabla 1, muestra algunas otras dimensiones del DO identificadas por los autores.

Tabla 1 Dimensiones que caracterizan al DO en instituciones educativas

\begin{tabular}{|c|c|c|}
\hline No. & Dimensiones & Autor(es) \\
\hline 1 & Mejora de procesos & (Leitner, 2004; López, 2010; Maldonado, 2000) \\
\hline 2 & Innovación & $\begin{array}{l}\text { (Campos,1998; Fazlagic, 2005; Millán y Díaz, 2010; Pérez y Pino, } \\
\text { 2018) }\end{array}$ \\
\hline 3 & Productividad & (Leitner, 2004; López, 2010; Maldonado, 2000) \\
\hline 4 & Calidad & (Leitner, 2004; Maldonado, 2000) \\
\hline 5 & Planes curriculares & $\begin{array}{l}\text { (Agasisti et al., 2016; Bueno et al., 2008; Kelly, 2004; Leitner, 2004; } \\
\text { López, 2010; Sánchez et al., 2009; Vidal, 2017) }\end{array}$ \\
\hline 6 & Infraestructura & $\begin{array}{l}\text { (Agasisti et al., 2016; Bueno et al., 2008; Kelly, 2004; Leitner, 2004; } \\
\text { Sánchez et al., 2009; Vidal, 2017) }\end{array}$ \\
\hline 7 & $\begin{array}{c}\text { Clima escolar y } \\
\text { liderazgo }\end{array}$ & (López, 2010) \\
\hline 8 & Gestión de recursos & $\begin{array}{l}\text { (Berg y Pinheiro, 2016; Hossain, 2018; Leitner, 2004; López, 2010; } \\
\text { Maldonado, 2000; Millán y Díaz, 2010; Moreno, 2017; Ploom y } \\
\text { Haldma, 2013) }\end{array}$ \\
\hline 9 & $\begin{array}{l}\text { Responsabilidad } \\
\text { educativa }\end{array}$ & $\begin{array}{l}\text { (Berg y Pinheiro, 2016; Broucker et al., 2017; Hossain, 2018; } \\
\text { Leitner, 2004; Ploom y Haldma, 2013) }\end{array}$ \\
\hline
\end{tabular}

Fuente: Elaboración propia en base a autores citados.

Es importante mencionar que, en el caso de la gestión de recursos, algunos autores han apoyado la idea de medir el DO por medio de indicadores financieros (Ayuya et al., 2017; Gogan et al., 2016; Goh et al., 2012) y algunos otros consideran que los indicadores no financieros son los más adecuados (Bollen et al., 2005; Bontis, 1998; Delaney y Huselid, 1996; Ngo y Loi, 2008; Pirozzi y Ferulano, 2016; Vargas y Lloria, 2017; Wu y Fang, 2010).

Finalmente, la literatura expone algunas investigaciones que han medido el DO en instituciones educativas, tomando en cuenta aspectos como la capacidad escolar, gestión, procesos escolares, calidad educativa, entre otros y donde el nivel de las instituciones y los datos complejos provenientes de un sin número de fuentes (docentes, personal directivo, administrativo, de servicios, estudiantes, padres de familia, entre otros) marcan la pauta de los resultados obtenidos (Ali et al., 2012; Awan y Saeed, 2014; Coronel et al., 2018; López, 2010; Machorro y Romero, 2017; Ramírez-Zambrano et al., 2015). Así, esta investigación contempla al
DO como un concepto que integra aspectos relacionados con índices educativos logrados por medio de una gestión adecuada de recursos y procesos, una imagen institucional positiva con la interacción de actores institucionales internos y externos (Agasisti et al., 2016; Berg y Pinheiro, 2016; Bueno et al., 2008; Broucker et al., 2017; Hossain, 2018; López, 2010; Millán y Díaz, 2010; Moreno, 2017; Pérez y Pino, 2018; Ploom y Haldma, 2013; Sánchez et al., 2009; Vidal, 2017).

\subsection{El CH y su relación con el DO en instituciones educativas de EMS}

La permanencia y desarrollo del $\mathrm{CH}$ en las instituciones educativas ha representado un reto (Barbosa-Chacón et al., 2015), pues ha sido considerado como un elemento complicado de tratar. Sin embargo, se han generando modelos de estudio que centran al $\mathrm{CH}$ como un elemento capaz de influir positivamente en el DO (Leitner, 2004). En este sentido, Rondón y Ammar (2016), desarrollaron una propuesta de gestión del talento humano en donde el $\mathrm{CH}$ se fortalece al desarrollar sus capacidades; Toalá-Sánchez et al., (2017), generaron un modelo donde se 
identifica, administra y organiza el conocimiento a través de las habilidades de su $\mathrm{CH}$ y algunos otros autores han establecido modelos en donde presentan al $\mathrm{CH}$ como un eje central de sus estudios en las instituciones educativas (Bueno et al., 2004; Bustos et al., 2012; Leitner, 2004; Muñoz y Pérez, 2007; Žilvinas y Leitner, 2015).

\section{Tabla 2 Definiciones del $\mathrm{CH}$ en el ámbito educativo}

\begin{tabular}{|c|c|}
\hline Autor & Concepto \\
\hline Guzmán y Marín (2011) & $\begin{array}{l}\text { Elemento capaz de desarrollar tareas, ayudados de actitudes, } \\
\text { habilidades y conocimientos con una interacción social en el } \\
\text { contexto escolar }\end{array}$ \\
\hline Bahrami et al., (2013) & $\begin{array}{l}\text { Docentes, investigadores, estudiantes, administrativos entre otros } \\
\text { que cuenta con los conocimientos }\end{array}$ \\
\hline $\begin{array}{l}\text { Leitner (2004); Orozco } \\
\text { (2014); Farah y Abouzeid } \\
\text { (2017) }\end{array}$ & $\begin{array}{l}\text { Recurso más valioso donde se genera el conocimiento en las } \\
\text { instituciones escolares }\end{array}$ \\
\hline $\begin{array}{l}\text { Cheng (2015); Ramírez y } \\
\text { Gordillo (2014) }\end{array}$ & $\begin{array}{l}\text { Capital interno de la institución que desarrolla: valores, cultura, } \\
\text { políticas, procesos, patentes e innovaciones en sinergia con su } \\
\text { capital externo (reputación, imagen y relaciones) }\end{array}$ \\
\hline Hili et al., (2017) & $\begin{array}{l}\text { Es quien cuenta con el potencial para que, a través de su } \\
\text { experiencia, capacidad, habilidad, creatividad e innovación, } \\
\text { mejoren el desempeño institucional }\end{array}$ \\
\hline
\end{tabular}

Fuente: elaboración propia a partir de autores citados y Barreto y Azeglio (2013).

Relacionando los conceptos de la tabla

2, existen estudios empíricos que han identificado relaciones significativas entre el CH y el DO (Allui y Sahni, 2016; Ayaz et al., 2018; Barreto y Azeglio, 2013; GonzálezLoureiro y Teixeira, 2015); como por ejemplo, la investigación de Hili, et al., (2017), quienes evaluaron la influencia de los conocimientos del $\mathrm{CH}$ sobre el DO, encontrando efectos positivos; también, Bermúdez y Bravo (2016), consideraron a la formación como una de las dimensiones más débiles y necesarias de reforzar; además, Castro (2015), MartínezChairez y Guevara-Araiza (2015) y Xu et al., (2011), han realizado estudios en el tema buscando medir el impacto del $\mathrm{CH}$ en el DO educativo, a través de dimensiones como: práctica docente, experiencia, liderazgo, entre otros. Por su parte, la investigación de Villagrán et al., (2018), da cuenta que son las habilidades docentes, aquellas que aportan significativamente en el DO educativo; en el tema, Siu (2020) señala que estas aportaron hasta el $70 \%$ al desarrollo educativo de los planteles donde realizaron su investigación; además, Ayaz et al., (2018), Kane et al., (2011) y Reyes (2016) encontraron resultados
Así mismo, Castro (2015), considera que el obstáculo que ha impedido la generación clara de investigaciones en el tema, así como un concepto generalizado, ha sido la fuerte dependencia del sector con el Estado; la tabla 2, muestra algunas definiciones establecidas por los autores. similares.

El trabajo realizado por Lora et al., (2018), al analizar la dimensión competencia del $\mathrm{CH}$ en el contexto, muestra que esta aporta un $77 \%$ a la formación de su $\mathrm{CH}$ y como resultado el DO educativo se ve beneficiado. Morales et al., (2017), González (2018) y Fernándes et al., (2016), estudiando aspectos como trabajo en equipo, gestión del aprendizaje, entre otras, han encontrado resultados semejantes.

Por último, es importante destacar que, a diferencia de las investigaciones descritas, Pérez et al., (2005) encontraron una relación negativa entre el $\mathrm{CH}$ y el $\mathrm{DO}$ en su estudio, al igual que Decker et al., (2004), quien, en su momento, determinó resultados poco claros en su investigación.

Derivado de lo anterior, es posible establecer la hipótesis de la presente investigación:

HI. Los factores determinantes del $\mathrm{CH}$, tienen un efecto positivo y significativo en el DO, en las instituciones tecnológicas de educación media superior 


\section{METODOLOGÍA}

\subsection{Técnica de recopilación de datos}

Tomando en cuenta investigaciones previas relacionadas con el $\mathrm{CH}$ y el $\mathrm{DO}$ en instituciones educativas, así como una retroalimentación pertinente de expertos (Lloret-Segura et al., 2014; Gil et al., 2000), se elaboró un cuestionario estructurado en tres secciones. La primera con 18 ítems y con una escala tipo Likert de cinco puntos: 1. Nunca, 2. Rara vez, 3. Algunas veces, 4. La mayoría de las veces, 5. Siempre, se enfocó en la recolección de datos relacionados con el $\mathrm{CH}$.

La sección dos, se integró de 23 reactivos referentes al DO dentro de los planteles, aplicando también la escala de cinco puntos. Finalmente, la sección tres recabó datos generales del encuestado (puesto, antigüedad, edad, escolaridad, género), de su plantel y zona geográfica donde se ubica.

\subsection{Muestra y estrategia de recolección de datos}

El estudio cuantitativo, presenta un diseño no experimental y un alcance explicativo. El trabajo de campo, se realizó en el estado de Tamaulipas, durante el mes de septiembre del 2020; sin embargo, es importante destacar que debido a la pandemia del COVID-19, la recolección de los datos se organizó mediante la colaboración de la coordinación estatal a nivel EMS tecnológica, quien, por medio de las academias escolares hizo llegar la liga electrónica para que los docentes de los 23 planteles respondieran por medio on line.
En este sentido, la población total en dichos centros educativos es de 2280 docentes, por lo que, al aplicar la fórmula de poblaciones finitas, con un nivel de confianza del $95 \%$ y un margen de error del 5\%, el tamaño de muestra apropiado indica 329 individuos (Münch y Ángeles, 2009). Así, se recopilaron 300 instrumentos, representado una tasa de respuesta del $91 \%$ sobre el tamaño de muestra determinado $(n=329)$.

\subsection{Técnicas de análisis de los datos}

Se realizó un análisis exploratorio de datos (AED) donde a través de la estadística descriptiva se estudió la muestra no probabilística conformada $(n=300)$. Dentro de la segunda fase del análisis y al tratarse de un instrumento elaborado para la particularidad del contexto, se exploró mediante el análisis factorial exploratorio (AFE) la estructura subyacente de las variables latentes, esperando un KMO igual o superior a 0.70 y $\mathrm{CF}$ superiores a 0.45 (Hair et al., 2014; Manzano, 2018; Pedraza, 2020; Pérez y Medrano, 2010).

Como tercera etapa, para efecto de valorar las hipótesis de investigación, se usó el modelo de ecuaciones estructurales (SEM) mediante la técnica de mínimos cuadrados parciales (PLS) por medio del sistema SmartPLS; los criterios de confiabilidad, validez convergente y validez discriminante tomados en cuenta (Martínez y Fierro, 2018; Pedraza, 2020; Rosseel, 2012), se encuentran referidos en la tabla 3 con sus parámetros de medición correspondiente.

Tabla 3 Criterios de validación del modelo de medida

\begin{tabular}{|c|c|c|c|}
\hline \multicolumn{2}{|c|}{ Indicador } & \multirow[t]{2}{*}{ Parámetro } & Autor(es) \\
\hline \multirow{4}{*}{ Confiablidad } & $\mathrm{CF}$ & & $\begin{array}{l}\text { (Araúz, 2015; Chin, 1998; Garson, 2013; } \\
\text { Ghozali, 2014; Hair et al., 2014; Lloret-Segura } \\
\text { et al., 2014; Martínez y Sepúlveda, 2012) }\end{array}$ \\
\hline & $\begin{array}{l}\text { Alfa de } \\
\text { Cronbach }\end{array}$ & $\begin{array}{l}>0.80 \\
>0.60 *\end{array}$ & $\begin{array}{l}\text { (González y Pazmiño, 2015; Hashim et al., } \\
\text { 2015) }\end{array}$ \\
\hline & $\begin{array}{l}\text { Fiabilidad } \\
\text { compuesta }\end{array}$ & $>0.70$ & (Araúz, 2015; Henseler, 2017; Hair et al., 2014) \\
\hline & RhoA & $>0.70$ & (Henseler, 2017) \\
\hline $\begin{array}{c}\text { Validez } \\
\text { Convergente }\end{array}$ & AVE & $>0.50$ & (Araúz, 2015; Chin, 1998) \\
\hline $\begin{array}{l}\text { Validez } \\
\text { Discriminante }\end{array}$ & $\begin{array}{l}\text { Fornell } \\
\text { Larcker }\end{array}$ & $\begin{array}{l}\text { La raíz cuadrada del AVE de } \\
\text { cada variable latente debe ser } \\
\text { mayor que las correlaciones que } \\
\text { tiene éste con el resto de las } \\
\text { variables, observándose ello en }\end{array}$ & $\begin{array}{l}\text { (Araúz, 2015; Hair et al., 2014; Henseler, } 2017 \\
\text { Henseler et al., 2015) }\end{array}$ \\
\hline
\end{tabular}




\begin{tabular}{cll}
\hline Indicador & \multicolumn{1}{c}{ Parámetro } & Autor(es) \\
\hline & la diagonal de la matriz de \\
& validez. \\
Cargas & La carga del indicador debe ser \\
Cruzadas & la mas alta en el factor \\
& categorizado y no en los demás \\
\hline
\end{tabular}

NOTA: CF: Carga factorial. *Cuando la teoría es escasa. AVE: Varianza media extraída

Fuente: elaboración propia

Así, para evaluar las hipótesis de investigación (estructura), se consideró la significancia estadística (bootstraping) con el estadístico $\mathrm{T}$, esperando valores $>1.96$ y donde el $\mathrm{R}^{2}$ (relevancia de precisión en la predicción del modelo) esté cercano a la unidad (Hair et al., 2014; Hair et al., 2016). Finalmente, para medir el efecto de la variable independiente (CH) sobre la dependiente (DO) se observa el $\mathrm{f}^{2}$, esperando un indicador superior a 0.15 (Hair et al., 2016), un $\mathrm{q}^{2}$ positivo y un indicador SRMR, que mide la diferencia entre la matriz de correlación observada y la de correlaciones implícitas del modelo, con índices menores a 0.08; por último, se analizó el NFI donde se esperaron valores mayores o iguales a 0.90 (Hair et al., 2016).

La siguiente sección muestra los resultados de este estudio.

\section{RESULTADOS}

\subsection{Los encuestados y su caracterización}

De los 300 instrumentos recopilados, el $30.7 \%$ (92 docentes) es personal que cuenta con un rango de 0 a 5 años de antigüedad en su puesto, seguidos por 48 empleados que se posicionan en el rango de más de 31 años de antigüedad laboral dentro del sistema y aquellos que cuentan con entre 25 y 30 años laborales (14.3\%). Así mismo, 126 docentes indicaron tener más de 50 años de edad, seguidos por el rango de 34 a 41 años, el cual se conforma por 70 trabajadores. También, el 55\% de la muestra indicó contar con grado de licenciatura, seguido por aquellos que tienen maestría como su último grado de estudios $(40.3 \%)$.

Adicionalmente, el porcentaje de participación entre hombres y mujeres para el cuestionario ha sido equilibrado, el $49 \%$ corresponde a varones y el $51 \%$ es el obtenido por docentes femeninas $\left(\begin{array}{llll}147 & \text { y } & 153\end{array}\right.$ respectivamente). Finalmente, la variable: zona, nos muestra información de los planteles donde los encuestados participaron más activamente, aquellos ubicados en la región norte del estado han sido los más representativos dentro del estudio (42.3\%), seguida por la zona sur $(31.7 \%)$ y centro del estado (26\%).

4.2. Análisis factorial exploratorio de las variables de análisis (CH y DO)

De acuerdo al AFE (Ferrando y Lorenzo-Seva, 2014; Lloret-Segura et al., 2014; Gil et al., 2000) en la variable $\mathrm{CH}$ se identificaron tres factores, los cuales han sido nombrados de acuerdo a la naturaleza de los reactivos que integraron como: competencias generales, competencias docentes y actitudes y valores. Así, aquellos que contaron con las cargas factoriales (CF) adecuadas (Chin, 1998; Garson, 2013; Ghozali, 2014; Hair et al., 2014; Lloret-Segura et al., 2014; Martínez y Sepúlveda, 2012) han sido 15 (de 18 originales), mismos que se detallan en la tabla 4.

Tabla 4 Factores determinantes del $\mathrm{CH}$ y su caracterización

\begin{tabular}{|c|c|c|c|}
\hline Factor & $\mathbf{C F}$ & $\begin{array}{l}\text { Alpha de } \\
\text { Cronbach }\end{array}$ & $\begin{array}{l}\text { AFE con } \\
\text { SPSS }\end{array}$ \\
\hline Factor 1: Competencias generales & & & \\
\hline $\begin{array}{l}\text { CH2. Recibe capacitación oportuna y de calidad que puede aplicar de } \\
\text { inmediato a sus funciones }\end{array}$ & 0.584 & & \\
\hline $\begin{array}{l}\text { CH3. Usa eficientemente el tiempo para el cumplimiento de objetivos y } \\
\text { metas en sus funciones }\end{array}$ & 0.883 & 0.966 & $\begin{array}{c}0.970 \\
\mathbf{C h i}^{2}\end{array}$ \\
\hline $\begin{array}{l}\text { CH4. Propicia y usa eficientemente el material educativo en el proceso de } \\
\text { enseñanza-aprendizaje }\end{array}$ & 0.787 & & $\begin{array}{l}4151.311 \\
\text { gl. } 105\end{array}$ \\
\hline CH5. Logra transmitir el conocimiento adecuadamente a fin de mejorar el & 0.604 & & Sig. .000 \\
\hline
\end{tabular}




\begin{tabular}{|c|c|c|c|}
\hline Factor & $\mathbf{C F}$ & $\begin{array}{l}\text { Alpha de } \\
\text { Cronbach }\end{array}$ & $\begin{array}{l}\text { AFE con } \\
\text { SPSS }\end{array}$ \\
\hline \multicolumn{4}{|l|}{ rendimiento de los estudiantes } \\
\hline $\begin{array}{l}\mathrm{CH} 7 \text {. Participa activamente en la elaboración de planeaciones curriculares, } \\
\text { proyectos educativos, eventos escolares, entre otros. }\end{array}$ & 0.680 & & \\
\hline $\begin{array}{l}\text { CH9. Desarrolla estrategias de aprendizajes significativos a fin de } \\
\text { promover la participación activa y creativa de los estudiantes }\end{array}$ & 0.644 & & \\
\hline $\begin{array}{l}\text { CH14. Son pacientes y perseverantes en la solución de problemas, logro } \\
\text { de objetivos y metas propuestas por la institución }\end{array}$ & 0.467 & & \\
\hline \multicolumn{4}{|l|}{ Factor 2: Competencias docentes } \\
\hline CH8. Trabaja con un enfoque de resolución de problemas & 0.596 & & \\
\hline $\begin{array}{l}\text { CH10. Implementa diversas técnicas e instrumentos de evaluación a fin de } \\
\text { medir los aprendizajes adquiridos conociendo las características de sus } \\
\text { estudiantes }\end{array}$ & 0.734 & & \\
\hline $\begin{array}{l}\text { CH11. Implementa estrategias de enseñanza-aprendizaje con participación } \\
\text { activa del entorno }\end{array}$ & 0.857 & & \\
\hline $\begin{array}{l}\text { CH12. Toma en cuenta las necesidades e intereses de sus estudiantes al } \\
\text { elaborar sus sesiones de trabajo }\end{array}$ & 0.680 & & \\
\hline $\begin{array}{l}\mathrm{CH} 13 \text {. Absorbe datos, información y conocimiento de su contexto a fin de } \\
\text { adaptarlo oportuna y eficazmente a sus funciones }\end{array}$ & 0.508 & & \\
\hline \multicolumn{4}{|l|}{ Factor 3: Actitudes y Valores } \\
\hline CH16. Respeta la heterogeneidad de sus miembros en todos los contextos & 0.873 & & \\
\hline $\begin{array}{l}\text { CH17. Muestra en general una actitud positiva frente a las innovaciones } \\
\text { educativas y directrices pedagógicas y administrativas }\end{array}$ & 0.649 & & \\
\hline $\begin{array}{l}\text { CH18. Considera que los logros no alcanzados son una oportunidad para } \\
\text { seguir aprendiendo }\end{array}$ & 0.720 & & \\
\hline
\end{tabular}

Nota: $\mathrm{CF}=$ carga factorial

Fuente: elaboración propia

De la misma forma, en la tabla 4 , se expone la confiabilidad a través del Alpha de Cronbach y el KMO, en donde se presentan valores adecuados superiores a 0.70 (González y Pazmiño, 2015; Hashim et al., 2015). Con ello, las competencias generales, como un factor del $\mathrm{CH}$, coinciden con investigaciones como la de Bermúdez y Bravo (2016), Hili et al., (2017) y Martínez-Chairez y GuevaraAraiza (2015); también, autores como Morales et al., (2017), González (2018), Fernándes et al., (2016) y Lora et al., (2018), estudiaron la competencia docente como dimensión del $\mathrm{CH}$ y en general señalaron a esta dimensión, como

un elemento valioso institucionalmente. Así mismo, se han realizado investigaciones analizado el $\mathrm{CH}$ a través de actitudes, valores, sentimientos y creencias, identificándolo como un elemento clave en la mejora del DO (Zlate y Enache, 2015), aunque Cuentas (2018) mencione un trabajo insuficiente en el tema.

En el caso del constructo DO, se ha generado un solo factor integrado por los 23 reactivos originales, los cuales han cumplido con las CF correspondientes y con un adecuado Alpha de Cronbach (tabla 5).

Tabla 5 Factor determinante del DO y su caracterización

\begin{tabular}{|c|c|c|c|}
\hline Desempeño Organizacional & CF & $\begin{array}{l}\text { Alpha de } \\
\text { Cronbach }\end{array}$ & $\begin{array}{l}\text { AFE con } \\
\text { SPSS }\end{array}$ \\
\hline DO1. Se cuenta con bajas tasas de deserción estudiantil & 0.791 & \multirow{5}{*}{0.979} & KMO \\
\hline $\begin{array}{l}\text { DO2. Existe diversidad y efectividad en las técnicas de enseñanza que se } \\
\text { aplican }\end{array}$ & 0.825 & & $\begin{array}{c}0.973 \\
\text { Chi }^{2}\end{array}$ \\
\hline DO3. Se cuenta con bajo ausentismo laboral & 0.723 & & 7745.804 \\
\hline $\begin{array}{l}\text { DO4. Se aplican de forma ágil los nuevos conocimientos a fin de mejorar } \\
\text { los procesos y el servicio ofrecido }\end{array}$ & 0.798 & & $\begin{array}{l}\text { gl. } 253 \\
\text { Sig. } .000\end{array}$ \\
\hline DO5. Se cuenta con integrantes certificados y a la vanguardia educativa & 0.763 & & \\
\hline
\end{tabular}




\begin{tabular}{|c|c|c|c|}
\hline Desempeño Organizacional & $\mathbf{C F}$ & $\begin{array}{l}\text { Alpha de } \\
\text { Cronbach }\end{array}$ & $\begin{array}{c}\text { AFE con } \\
\text { SPSS }\end{array}$ \\
\hline DO6. Se cuenta con certificaciones que acrediten los procesos centrales & 0.802 & & \\
\hline $\begin{array}{l}\text { DO7. Se generan recursos a través de proyectos educativos (estatales y } \\
\text { federales) que favorecen al plantel }\end{array}$ & 0.801 & & \\
\hline DO8. En cada ciclo escolar, se incrementa el número de matrícula & 0.827 & & \\
\hline $\begin{array}{l}\text { DO9. Cuenta con recursos suficientes para financiar proyectos educativos } \\
\text { que benefician al plantel }\end{array}$ & 0.853 & & \\
\hline $\begin{array}{l}\text { DO10. Se gestionan los recursos a fin de atender equilibradamente las } \\
\text { actividades educativas, administrativas y de apoyo en el plantel } \\
\text { educativas, administrativas y de apoyo }\end{array}$ & 0.882 & & \\
\hline $\begin{array}{l}\text { DO11. Se cuenta con las condiciones adecuadas en infraestructura y } \\
\text { equipamiento para el uso y desarrollo óptimo de las funciones }\end{array}$ & 0.830 & & \\
\hline $\begin{array}{l}\text { DO12. Se generan mejoras en los procesos educativos otorgados en el } \\
\text { plantel }\end{array}$ & 0.905 & & \\
\hline $\begin{array}{l}\text { DO13. Los tiempos de repuesta para los procesos educacionales son } \\
\text { adecuados. }\end{array}$ & 0.812 & & \\
\hline $\begin{array}{l}\text { DO14. Los tiempos de repuesta para los procesos administrativos son } \\
\text { adecuados. }\end{array}$ & 0.883 & & \\
\hline $\begin{array}{l}\text { DO15. Se actualizan permanentemente los programas de estudio, de } \\
\text { trabajo, manuales, procedimientos, reglamentos, entre otros }\end{array}$ & 0.805 & & \\
\hline $\begin{array}{l}\text { DO16. Se adaptan rápidamente los procesos a fin de cubrir las } \\
\text { necesidades de sus integrantes, estudiantes y comunidad en general }\end{array}$ & 0.849 & & \\
\hline $\begin{array}{l}\text { DO17. Cada vez es mas frecuente que se realicen innovaciones (Uso de } \\
\text { bases de datos, generación de aplicaciones, proyectos educativos, entre } \\
\text { otros) que mejoran el desempeño del plantel }\end{array}$ & 0.885 & & \\
\hline $\begin{array}{l}\text { DO18. Los estudiantes están satisfechos con la formación académica } \\
\text { generada a raíz de la formación, habilidades, competencia, compromiso, } \\
\text { actitudes y valores del capital humano que conforma el plantel }\end{array}$ & 0.850 & & \\
\hline $\begin{array}{l}\text { DO19. El sector productivo esta satisfecho con la calidad académica de } \\
\text { los egresados lo que se refleja en una tasa alta de empleabilidad }\end{array}$ & 0.835 & & \\
\hline $\begin{array}{l}\text { DO20. Los egresados se colocan fácilmente en el nivel superior puesto } \\
\text { que se percibe una adecuada formación de los mismos }\end{array}$ & 0.823 & & \\
\hline $\begin{array}{l}\text { DO21. Genera una buena imagen y frecuentemente excede las } \\
\text { expectativas de los padres de familia, egresados, sociedad y autoridades } \\
\text { estatales y federales }\end{array}$ & 0.830 & & \\
\hline $\begin{array}{l}\text { DO22. Se acude con frecuencia a exposiciones de proyectos educativos } \\
\text { tanto local, estatal como nacionalmente }\end{array}$ & 0.802 & & \\
\hline $\begin{array}{l}\text { DO23. Se impulsan acuerdos de colaboración con otros planteles o } \\
\text { empresas a fin de mejorar los procesos institucionales }\end{array}$ & 0.791 & & \\
\hline
\end{tabular}

Nota: $\mathrm{CF}=$ carga factorial

Fuente: elaboración propia

También, el KMO arroja un índice de 0.973, lo cual es conveniente para esta investigación. Así, dentro del AFE se identificó a la variable $\mathrm{CH}$ con un comportamiento multidimensional, mientras que la variable de DO se conforma en un solo factor para su estudio.

Tabla 6 Indicadores de fiabilidad y validez convergente para el modelo mediante SEM-PLS

\begin{tabular}{ccc}
\hline $\begin{array}{c}\text { Fiabilidad } \\
\text { Compuesta }\end{array}$ & Constructo & $\begin{array}{c}\text { Varianza } \\
\text { media extraída } \\
\text { (AVE) }\end{array}$ \\
\hline 0.937 & Competencias generales & 0.713 \\
0.909 & Competencias docentes & 0.834 \\
\hline
\end{tabular}




\begin{tabular}{ccc}
\hline $\begin{array}{c}\text { Fiabilidad } \\
\text { Compuesta }\end{array}$ & Constructo & $\begin{array}{c}\text { Varianza } \\
\text { media extraída } \\
\text { (AVE) }\end{array}$ \\
\hline 0.922 & Actitudes y valores & 0.797 \\
0.956 & Desempeño organizacional & 0.662 \\
\hline
\end{tabular}

Fuente: elaboración propia, 2021.

También, para evaluar el modelo estructural (figura 3) se han empleado criterios como: la estimación de la significancia estadística e importancia de las hipótesis por medio del procedimiento Boostrapping (tabla

Figura 3 Modelo estructural de los factores CH y DO de los docentes en instituciones de EMS

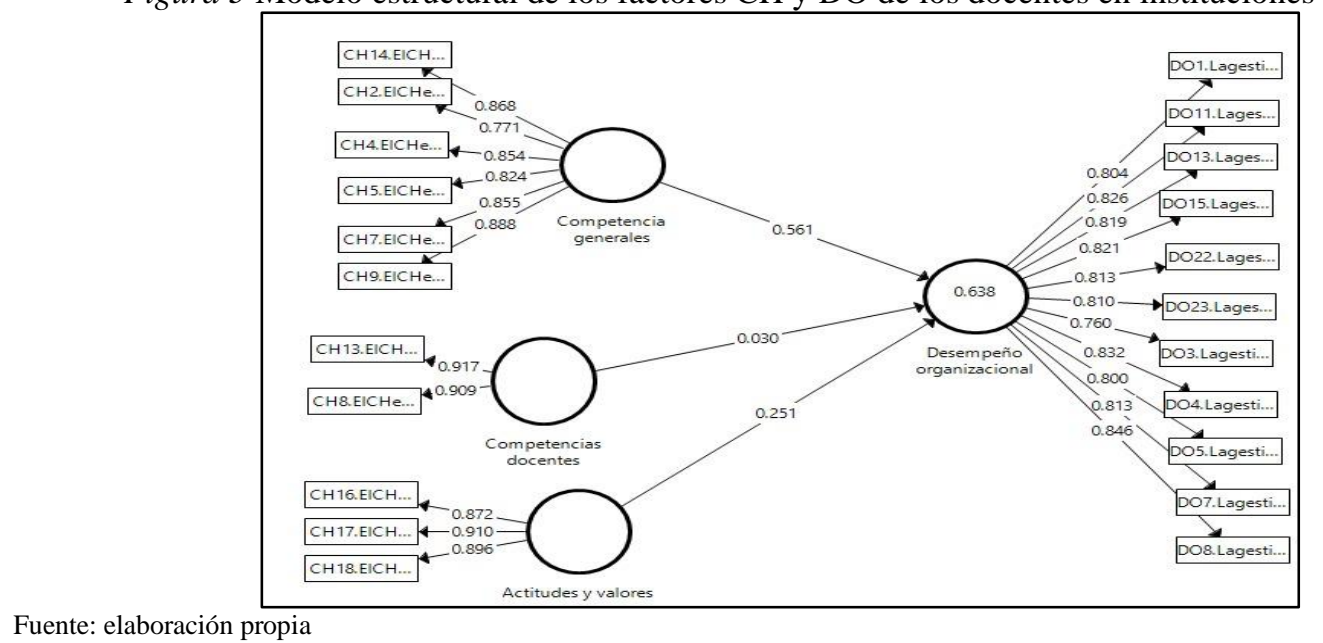

Fuente: elaboración propia

Tabla 7 Valores T y P para validar las hipótesis de investigación

\begin{tabular}{ccccl}
\hline Hipótesis & Relación & Valor & Valor & Hipótesis \\
\hline H1 & T & P & \multicolumn{1}{c}{ Se acepta } \\
H2 & Competencias generales - DO & 7.803 & 0.000 & Se acechaza \\
H3 & Competencias docentes - DO & 0.459 & 0.646 & Se rech \\
\hline
\end{tabular}

Fuente: elaboración propia. Valor $\mathrm{T}=\mathrm{valor}$ observado del estadístico de la prueba t, valor $\mathrm{P}=$ nivel de significancia mas bajo.

El resultado del estadístico T, permite

inferir la aceptación de dos de las tres hipótesis causales establecidas en el presente estudio. El $\mathrm{R}^{2}$ obtenido indica valores consistentes donde el DO se explica en un $63.4 \%$. También, para medir los efectos de la variable independiente (factores determinados para la variable $\mathrm{CH}$ ) sobre la dependiente (DO) se observa el $\mathrm{f}^{2}$, el dato arrojado muestra que los valores oscilan entre 0.01 y 0.227 (Hair et al., 2016), lo que muestra efectos significativos.

Además, a través del estadístico $\mathrm{Q}^{2}$, donde lo recomendable es obtener valores superiores a 0.15 , se arrojan valores adecuados de 0.414 para DO (Hair et al., 2016), lo cual manifiesta la relevancia del modelo (Hair et al., 2014). Por último, en cuanto a los indicadores SRMR y NFI, que miden la bondad de ajuste, se han obtenido valores de
0.052 y 0.867 respectivamente, lo cual indica resultados favorables en ambos casos.

\section{CONCLUSIONES}

Este estudio genera conocimiento empírico relacionando la variable $\mathrm{CH}$ y analizando sus efectos en el DO de instituciones educativas tecnológicas a nivel medio superior. Las tres dimensiones identificadas del $\mathrm{CH}$ : competencias generales, competencias docentes, así como el factor de actitudes y valores, caracterizan la variable y coinciden con los hallazgos identificados por autores consultados como: Castro (2015), Hili et al., (2017), Lora et al., (2018), Martínez-Chairez y Guevara-Araiza (2015) y Xu et al., (2011).

En este sentido, los docentes consideran imperiosa su formación continua y la adquisición de conocimientos a fin de potencializar su labor dentro de las 
instituciones tecnológicas educativas del sector, por ende, dicha dimensión tiene un efecto positivo y significativo en el DO de los planteles. Este hallazgo se asocia con los beneficios permanentes que los docentes reciben con programas de capacitación y formación educativa (llamadas jornadas de capacitación), en donde actualizan sus conocimientos para evaluar los aprendizajes, elaborar material didáctico, mejorar sus habilidades tecnológicas, entre otras.

Además, las actitudes y valores también exponen una relación positiva y significativa relacionada con el DO. En este aspecto, los resultados se asocian a que dentro del sistema, sus miembros comparten valores fuertemente arraigados a su contexto laboral, existen índices altos de asistencia e impartición de clase y también una participación activa en las convocatorias locales y nacionales en diversos concursos de conocimiento y tecnología; también, la caracterización del personal indica que el $30.7 \%$ de los mismos, son trabajadores de reciente ingreso al sistema y el $42 \%$ son docentes que cuentan con mas de 50 años de edad, ambas características denotarían actitudes y valores beneficiosos para el sistema. Este hallazgo se relaciona con el identificado por Cuentas (2018) y Zlate y Enache (2015) quienes consideraron aspectos como: compromiso, creencias y actitud positiva como elementos indispensables del factor.

Por otro lado, los datos indican que las competencias docentes no aportan al DO (rechazando la hipótesis establecida de esta relación); lo que se contrapone con los resultados generados por: Lora et al. (2018), González (2018), Cuentas (2018) y Morales (2017), autores consultados y quienes señalaron que las competencias adecuadas en las instituciones que estudiaron, permitieron orientar asertivamente el aprendizaje de los estudiantes y beneficiar en consecuencia el DO en las instituciones objeto de sus estudios. Entonces se infiere que, en esta investigación particular, un enfoque para resolver problemas, la implementación de estrategias didácticas y el uso de los conocimientos para el desarrollo de sus funciones, no son factores determinantes en los resultados obtenidos en el desempeño de los planteles.

Sin embargo, lo anterior es un hallazgo que detona una línea futura de investigación, a fin de comprender este fenómeno derivado de este resultado contradictorio; entonces estudios posteriores apoyados en entrevistas y observación, permitirían identificar las categorías mas pertinentes para su análisis, comprensión y efectos sobre los resultados educativos de estos planteles.

Como implicaciones del presente estudio, es importante destacar que los resultados obtenidos no pueden ser generalizados a todo el sistema tecnológico de EMS, pues los mismos, representan solo una participación limitada de estas instituciones. Así mismo, se ha desarrollado un modelo conceptual de investigación, estableciendo la relación entre el $\mathrm{CH}$ educativo y el DO, por lo que se ha contribuido en conocimiento empírico que marca la pauta para que, a nivel directivo se cuente con la información pertinente para tomar decisiones de gestión, educativas y administrativas adecuadas.

En cuanto a las futuras líneas de investigación, estudios posteriores podrían considerar variables relacionadas con el aprendizaje institucional, liderazgo, cultura, compromiso, entre otros; además sería conveniente el establecimiento de una muestra aleatoria heterogénea incluyendo personal directivo y administrativo a fin de enriquecer los estudios. 


\section{REFERENCIAS}

Agasisti, T., Barra, C., y Zotti, R. (2016). Evaluating the efficiency of Italian public universities (2008-2011) in presence of (unobserved) heterogeneity. Socio-Economic Planning Sciences, 55, 47-58.

Ali, K., Zohreh, S., y Khodadoost, R. (2012). Designing a model to recognize and manage intellectual capital in education system. Procedia-Social and Behavioral Sciences, 46, 992-997.

Allui, A., y Sahni, J. (2016). Strategic human resource management in higher education institutions: Empirical evidence from Saudi. Procedia-Social and Behavioral Sciences, 235, 361-371.

Aráuz, A. F. (2015). Aplicación del análisis factorial confirmatorio a un modelo de medición del rendimiento académico en lectura. Revista de Ciencias Económicas, 33(2), 39-65.

Araya-Castillo, L., y Rivera-Arroyo, J. (2021). ¿Cómo las instituciones de educación superior deben enfrentar los nuevos desafíos del entorno?. Revista de Ciencias Sociales (Ve), 27(1), 22-32.

Awan, A. G. y Saeed, K. (2014). Intellectual capital and research performance of universities in southern Punjab-Pakistan. European Journal of Business and Innovation Research, 2(6), 21-39

Ayaz, M., Qahar, A., Ullah, R. y Afeef, M. (2018). Assessing the Influence of Selected Human Capital Management Factors on the Organizational Performance of Higher Education Sector in Afghanistan. Journal of Business and Tourism, 4(02), 173-180

Ayuya, A. M., Awino, Z. B., Machuki, V. N., y Wainaina, G. (2017). Strategic choice and performance of universities in Kenya: The mediating role of organizational learning. $D B A$ Africa Management Review, 7(2).

Bahrami, S., Rajaeepour, S., Yarmohmmadian, M., y BakhtiarNarsabadi, H. (2013). Simple and multiple relations between strategic human resource management and intellectual capital in Iranian higher education. African Journal of Business Management, 7(35), 3451-3460. http://doi.org/10.5897/AJBM12.104

Bak, O. (2012). Universities: can they be considered as learning organizations? A preliminary micro-level perspective. The Learning Organization, 19(2), 163-172. https://doi.org/10.1108/09696471211201515

Barbosa-Chacón, J. W., Barbosa Herrera, J. C., y Rodríguez Villabona, M. (2015). Concepto, enfoque y justificación de la sistematización de experiencias educativas: Una mirada" desde" y" para" el contexto de la formación universitaria. Perfiles educativos, 37(149), 130-149.

Barreto, A., y Azeglio, A. (2013). La problemática de la gestión del capital humano en las MiPymes de alojamiento turístico de la ciudad de Buenos Aires- Argentina. Estudios y perspectivas en turismo, 22(6), 1140-1159. http://www.scielo.org.ar/scielo.php?pid=S185117322013000600007\&script=sci_arttext

Berg, L. N., y Pinheiro, R. (2016). Handling different institutional logics in the public sector: Comparing management in Norwegian universities and hospitals. In Towards a comparative institutionalism: Forms, dynamics and logics across the organizational fields of health care and higher education. Emerald Group Publishing Limited.

Bermúdez, E. y Bravo, V. (2016). Habilidades directivas y desempeño laboral del personal docente en escuelas básicas primarias. Omnia, 22(3),60-70. https://www.redalyc.org/articulo.oa?id=737/73752819005

Bollen, L., Vergauwen, P. y Schnieders, S. (2005). Linking Intellectual Capital and Intellectual Property to Company Performance. Management Decision, 43(9), 1161-1185. https://doi.org10.1108/ 00251740510626254

Bontis, N. (1998). Intellectual capital: An exploratory study that develops measure and models. Management Decision, 2(36), 63-76. http://doi.org/10.1108/00251749810204142

Broucker, B., De Wit, K., y Verhoeven, J. (2017). Higher Education Research: Looking Beyond 
New Public Management. In Theory and Method in Higher Education Research, 3, $21-$ 38. https://doi.org/10.1108/S2056-375220170000003002

Bueno, E., Salmador, M. P., y Rodríguez, Ó. (2004). The role of social capital in today's economy. Journal of intellectual capital.

Bueno, E., Salmador, M. P., y Merino, C. (2008). Génesis, concepto y desarrollo del capital intelectual en la economía del conocimiento: Una reflexión sobre el Modelo Intellectus y sus aplicaciones. Estudios de economía aplicada, 26(2), 43-63.

Bustos, E., Morán, L., Álvarez, S., y Alemán, E. (2012). Gestión del capital intelectual para generar productividad científica en Instituciones de Educación Superior Públicas. Docencia e Innovación Tecnológicas, 10, 17-20.

Cabrol, M., y Székely, M. (2012). Educación para la transformación. BID

Campos, E. B. (1998). El capital intangible como clave estratégica en la competencia actual. Boletín de estudios económicos, 53, 207.

Castro, A. (2015). Competencias administrativas y académicas en el profesorado de educación media superior. Revista mexicana de investigación educativa, 20(64), 263-294.

Chatterji, N., y Kiran, R. (2017). Role of human and relational capital of universities as underpinnings of a knowledge economy: A structural modelling perspective from north Indian universities. International Journal of Educational Development, 56, 52-61.

Cheng, E. (2015). Knowledge Sharing For Creating School Intellectual Capital. Procedia - Social and Behavioral $\quad$ Sciences, 191(852), 1455-1459. https://doi.org/10.1016/j.sbspro.2015.04.307

Chin, W. W. (1998). The partial least squares approach to structural equation modeling. Modern methods for business research, 295(2), 295-336.

Coronel, N. Y., Benguría, C. R., Rodríguez, C. P. C., Orozco, M. C., y Patiño-Ortiz, J. (2018). Propuesta de un modelo para mejorar el desempeño educativo del nivel Medio Superior del Instituto Politécnico Nacional. Científica, 22(1), 17-27.

Cuentas Martel, S. C. (2018). La gestión del capital humano en una unidad académica de educación superior: analysis of a university. Educación, 27(53), 57-72.

Decker, P. T., Mayer, D. P., y Glazerman, S. (2004). The effects of Teach for America on students: Findings from a national evaluation. University of Wisconsin--Madison, Institute for Research on Poverty.

Delaney, J. T., y Huselid, M. A. (1996). The impact of human resource management practices on perceptions of organizational performance. Academy of Management journal, 39(4), 949969.

Farah, A., y Abouzeid, S. (2017). The impact of intellectual capital on performance: Evidence from the public sector. Knowledge Management \& E-Learning: An International Journal, 9(2), 225-238.

Fazlagic, A. (2005). Measuring the intellectual capital of a university. Trabajo presentado en Trends in the management of human resources in higher education. París, Francia.

Fernándes, D. J., Sotolongo, M., y Martínez, C. C. (2016). La evaluación del desempeño por competencias: percepciones de docentes y estudiantes en la educación superior. Formación universitaria, 9(5), 15-24.

Ferrando, P. J., y Lorenzo-Seva, U. (2014). El análisis factorial exploratorio de los ítems: algunas consideraciones adicionales. Anales De Psicología/Annals of Psychology, 30(3), 11701175.

Garson, G. D. (2013). Two-Stage Least Square Regression. Statistical Associates Publishers.

Ghozali, I. (2014). SEM Metode Alternatif dengan menggunakan Partial Least Squares (PLS). Semarang: Badan Penerbit Universitas Diponegoro.

Gil, J. A. P., Moscoso, S. C., y Rodríguez, R. M. (2000). Validez de constructo: el uso de análisis factorial exploratorio-confirmatorio para obtener evidencias de validez. Psicothema, 12(Su2), 442-446. 
Gogan, L., Artene, A., Sarca, I., y Draghici, A. (2016). The Impact of Intellectual Capital on Organizational Performance. Procedia-Social and Behavioral Sciences, 221, 194-202. https://doi.org.10.1016/j. sbspro.2016.05.106

Goh, S. C., Elliott, C., y Quon, T. K. (2012). The relationship between learning capability and organizational performance. The learning organization, 19(2), 92-108.

González, J., y Pazmiño, M. (2015). Cálculo e interpretación del Alfa de Cronbach para el caso de validación de la consistencia interna de un cuestionario, con dos posibles escalas tipo Likert. Revista publicando, 2(1), 62-67.

González, M. O. (2018). Percepción del desempeño docente-estudiante en la modalidad mixta desde una mirada ecosistémica. RIDE. Revista Iberoamericana para la Investigación y el Desarrollo Educativo, 8(16), 370-393.

González-Loureiro, M., y Teixeira, A. M. (2015). Intellectual capital in public universities: a performance-oriented approach to manage intangible. International Journal of Engineering and Industrial Management, (3), 95-125.

Guthrie, J., y Dumay, J. (2015). New frontiers in the use of intellectual capital in the public sector. Journal of Intellectual Capital, 16(2), 258-266. http://doi.org/10.1108/JIC-02-2015-0017

Guzmán, I., y Marin, R. (2011). La competencia y las competencias docentes: reflexiones sobre el concepto y la evaluación. Revista electrónica interuniversitaria de formación del profesorado, 14(1), 151-163. https://doi.org/http://www.redalyc.org/articulo.oa?id=217017192012

Hair, J., Anderson, R., Barry, B. y Black, W. (2014). Multivariate data analysis, 7th Edition. Edinburg. Pearson Education Limited

Hair Jr., J. F., Hult, G. T. M., Ringle, C., y Sarstedt, M. (2016). A primer on partial least squares structural equation modeling (PLS-SEM). Sage publications.

Hashim, H., Ishak, N. A., y Hilmi, Z. A. G. (2015). Influence of organizational climate on disabled job embeddedness. Procedia-Social and Behavioral Sciences, 202, 242-251.

Henseler, J., Ringle, C. M., y Sarstedt, M. (2015). A new criterion for assessing discriminant validity in variance-based structural equation modeling. Journal of the academy of marketing science, 43(1), 115-135.

Henseler, J. (2017). Partial least squares path modeling. In Advanced methods for modeling markets (pp. 361-381). Springer, Cham.

Hili, P. Gani, M. U., Hamzah, N., Rahman, Z., y Sjahruddin, H. (2017). Effect of human capital and leadership on institutions performance and competitive advantages IRA-International. Journal of Management \& Social Sciences, 7(3), 479-488. http://dx.doi.org/10.21013/jmss.v7.n3.p9

Hossain, M. (2018). The Effects of Governance Reforms on School Supervision: An Analysis of Six Developing and Emerging Economies. Cross-nationally Comparative, Evidencebased Educational Policymaking and Reform. International Perspectives on Education and Society, 35, 127-160. https://doi.org/10.1108/S1479-367920180000035011

Kane, T. J., Taylor, E. S., Tyler, J. H., y Wooten, A. L. (2011). Identifying effective classroom practices using student achievement data. Journal of human Resources, 46(3), 587-613.

Kelly, A. (2004). The intellectual capital of schools: Analysing government policy statements on school improvement in light of a new theorization. Journal of Education Policy, 19(5), 609-629.

Kerlinger, F. y Lee, H. (2002). Investigación del comportamiento. 4ta. Edición, México. Ed. McGraw-Hill

Kline, R. (2011). Principles and Practice of Structural Equation Modeling (3a ed.). New York: The Guilford Press.

Leitner, K. H. (2004). Intellectual capital reporting for universities: conceptual background and application for Austrian universities. Research evaluation, 13(2), 129-140.

Lloret-Segura, S., Ferreres-Traver, A., Hernández-Baeza, A., y Tomás-Marco, I. (2014). El análisis 
factorial exploratorio de los ítems: una guía práctica, revisada y actualizada. Anales de Psicología/Annals of Psychology, 30(3), 1151-1169.

López, S. (2010). Cuerpos académicos: factores de integración y producción de conocimiento. Revista de la educación superior, 39(155), 7-25.

López, S. (2016). Competitividad de la educación superior en cuatro países de América Latina: perspectiva desde un ranking mundial. Revista de la educación superior, 45(178), 45-59.

Lora, L. E., Cruz, P. B., y Larios, B. I. O. (2018). Detección de variables predictoras de éxito y desempeño desfavorable de la actividad docente en pregrado presencial en una institución pública estatal. Edetania Estudios y propuestas socioeducativas, (54), 73-92.

Machorro, F., y Romero, M. V. (2017). Propuesta de un Instrumento de Autoevaluación del Desempeño Organizacional en Instituciones Públicas de Educación Superior en México. Formación universitaria, 10(3), 03-10.

Maldonado, A. (2000). Los organismos internacionales y la educación en México: El caso de la educación superior y el Banco Mundial. Perfiles educativos, 22(87), 51-75.

Manzano, A. P. (2018). Introducción a los modelos de ecuaciones estructurales. Investigación en educación médica, 7(25), 67-72.

Martínez, M., y Fierro, E. (2018). Aplicação da técnica PLS-SEM na gestão do conhecimento: uma abordagem técnica prática. RIDE. Revista Iberoamericana para la Investigación y el Desarrollo Educativo, 8(16), 130-164.

Martínez, C. M., y Sepúlveda, M. A. R. (2012). Introducción al análisis factorial exploratorio. Revista colombiana de psiquiatría, 41(1), 197-207.

Martínez-Chairez, G., y Guevara-Araiza, A. (2015). La evaluación del desempeño docente. Ra Ximhai, 11(4),113-124. https://www.redalyc.org/articulo.oa?id=461/46142596007

Mertens, P. (1979). Comparative Indicators for German Universities. International Journal of Institutional Management in Higher Education, 3(1), 155-68.

Millán, J. J. G., y Díaz, M. T. R. (2010). Modelos de Capital Intelectual y sus indicadores en la universidad pública. Cuadernos de administración, (43), 113-128.

Morales, R., Morales, R., y Yépez, M. J. B. (2017). Competencias docentes y desempeño: un estudio con profesores universitarios. Revista Publicando, 4(11), 248-269.

Moreno, C. I. (2017). Las reformas en la educación superior pública en México: rupturas y continuidades. Revista de la educación superior, 46(182), 27-44.

Muñoz, M. P. S., y Pérez, S. E. (2007). New management in higher education institutions: introducing intellectual capital approaches. Conradi Research Review, 4(2), 71.

Münch, L., y Ángeles, E. (2009). Métodos y técnicas de investigación.

Ngo, H. Y., y Loi, R. (2008). Human resource flexibility, organizational culture and firm performance: An investigation of multinational firms in Hong Kong. The International Journal of Human Resource Management, 19(9), 1654-1666.

OCDE (2018). OECD Labour force statistics 2017. Paris

OCDE (2017). Estudios Económicos de la OCDE. Organization for Economic Cooperation and Development

Orozco, F. C. E. (2014). Orientación de las organizaciones públicas al aprendizaje organizacional. El caso de los organismos descentralizados en el Estado de Sonora, México. Estudios gerenciales, 30(130), 10-17.

Pedraza, N. A. (2020). Organizational climate and job satisfaction of human capital: differentiated factors in public and private organizations. Innovar, 30(76), 9-23.

Pérez, E. R., y Medrano, L. A. (2010). Análisis factorial exploratorio: bases conceptuales y metodológicas. Revista Argentina de Ciencias del Comportamiento (RACC), 2(1), 58-66.

Pérez, S., Montes Peon, J. M., y Vazquez Ordas, C. J. (2005). Human resource practices, organizational learning and business performance. Human Resource Development International, 8(2), 147-164.

Pérez, O. G. B., y Pino, J. W. F. (2018). Rol de la gestión educativa estratégica en la gestión del 
conocimiento, la ciencia, la tecnología y la innovación en la educación superior. Educación Médica, 19(1), 51-55.

Pirozzi, M. y Ferulano, G. (2016). Intellectual Capital and Performance Measurement in Healthcare Organizations: An Integrated New Model. Journal of Intellectual Capital, 17(2), 320-350. http://doi.org. 10.1108/JIC-07-2015-0063

Ploom, K. y Haldma, T. (2013). Balanced performance management in the public education system. Baltic Journal of Management, 8(2), 183-207.

Ramírez, Y., y Gordillo, S. (2014). Recognition and measurement of intellectual capital in Spanish universities. Journal of Intellectual Capital, 15(1), 173-188. http://doi.org/10.1108/JIC05-2013-0058

Ramírez-Zambrano, A. A., Velasco-Arellanes, F. J., y Vera-Noriega, J. Á. (2015). Procesos Escolares y Eficiencia Interna: ¿Cuál es la Relación con el Logro Académico en la Educación Media Superior Mexicana? Education Policy Analysis Archives/Archivos Analíticos de Políticas Educativas, 23, 1-20.

Reyes, M. E. (2016). Relación entre Habilidades Sociales y Desempeño Docente desde la percepción de estudiantes adultos de universidad privada en Lima, Perú. Revista digital de investigación en docencia universitaria, 10(2), 17-31.

Rondón, M., y Ammar, M. (2016). Gestión del talento humano en organizaciones educativas. Revista de investigación, 40(88), 148-165.

Rosseel, Y. (2012). Lavaan: An R package for structural equation modeling and more. Version 0.512 (BETA). Journal of statistical software, 48(2), 1-36.

Sánchez, P., Elena S. y Castillo R., (2009). Intellectual capital dynamics in universities: a reporting model. Journal of intellectual capital, 10(2), 307-324. http://doi.org/ $10.1108 / 14691930910952687$

Schumacker, R. E., y Lomax, R. G. (2004). A beginner's guide to structural equation modeling. Psychology press.

Segatto, C. I., y Abrucio, F. L. (2017). A gestão por resultados na educação em quatro estados brasileiros. Revista do Serviço Público, 68(1), p. 85-106.

Segredo, A. M. (2016). Aproximación teórica a la evolución, teorías, enfoques y características que han sustentado el desarrollo de las organizaciones. Revista Cubana de Salud Pública, 42, $585-595$.

Siu, J. L. R. (2020). Las habilidades blandas como base del buen desempeño del docente universitario. INNOVA Research Journal, 5(2), 186-199.

Toalá-Sánchez, G., Meza-Bolaños, D., y Mejía-Madrid, G. (2017). Modelo de gestión del conocimiento y capital intelectual de los docentes universitarios. Revista Publicando, 4(11), 270-286.

Ullman, J. (2013). Structural equation modeling. In Barbara Tabachnick y Linda Fidell (Eds.), Using Multivariate Statistics, 681-785. Boston: Pearson.

Vargas, N., y Lloria, M. B. (2017). Performance and intellectual capital: how enablers drive value creation in organizations. Knowledge and Process Management, 24(2), 114-124

Vidal, C. R. (2017). Modelo de capital intelectual para la investigación en las universidades públicas de la Costa Caribe colombiana. Actualidades Investigativas en Educación, 17(1), 400427.

Villagrán, C., Hernández, M. E. M., y Delgado, S. C. (2018). Relación entre variables mediadoras del desempeño docente y resultados educativos: una aproximación al liderazgo escolar. Opción: Revista de Ciencias Humanas y Sociales, 87, 213-240

Wu, C. H., y Fang, K. (2010). Improving project performance through organizational learning: an empirical study in Taiwan. Technology Analysis \& Strategic Management, 22(2), 261276.

Wu, H. Y., Chen, J. K., Chen, I. S., y Zhuo, H. H. (2012). Ranking universities based on performance evaluation by a hybrid MCDM model. Measurement, 45(5), 856-880. 
Xu, Z., Hannaway, J., y Taylor, C. (2011). Making a difference? The effects of Teach for America in high school. Journal of policy Analysis and Management, 30(3), 447-469.

Žilvinas, G. S. S. E. P., y Leitner, M. K. H. (2015). An intellectual capital maturity model (ICMM) to improve strategic management in European universities: A dynamic approach. Journal Intellectual Capital, 16, 419-442.

Zlate, Ş., y Enache, C. (2015). The Interdependence Between Human Capital and Organizational Performance in Higher Education. Procedia - Social and Behavioral Sciences, 180, 136143. https://doi.org/10.1016/j.sbspro.2015.02.096 